\title{
Fruit flies help shed light on drug discovery for ALS
} incurable neurodegenerative disease that affects people in adulthood. It leads to the death of neurons involved in muscle control, eventually affecting almost all facets of the body, and breathing Drs Nancy Bonini and Leeanne McGurk at the using fruit flies, mammalian cellular systems like neurons, and in vitro protein preparation to investigate promising new molecules that could open avenues to new treatments
for this devastating condition.
Amyotrophic lateral sclerosis University of Pennsylvania are

$\bigwedge_{\begin{array}{c}\text { myotrophic lateral sclerosis (ALS) } \\ \text {-also known as motor neuron(e) } \\ \text { disease - is a severe and incurable }\end{array}}$ disease that has a devastating effect on the quality of life of people living with the condition as well as their carers and families. ALS most resonates with the general public in relation to high-profile personalities who have experienced the condion, player Lou Gehrig - hence, the disorder disease - and world-renowned British physicist, Stephen Hawking.

The first signs and symptoms of ALS might include tripping, leg weakness and difficulty controlling hand movements, or what people might first dismiss as simple clumsiness. Diagnosis is usually aided by imaging and scanning techniques that can rule out other causes of nerve damage and lack of motor control, including bran tumours and neuropathy. Over time, symptoms of ALS gradually become worse and affect almost all areas of the body causing muscle weakness, stiffness and paralysis. As the disease advances, becomes more difficult and eating becomes impossible as muscles asso with swallowing are affected. Almost their ability to speak frustratingly difficut leading some to rely on technology to

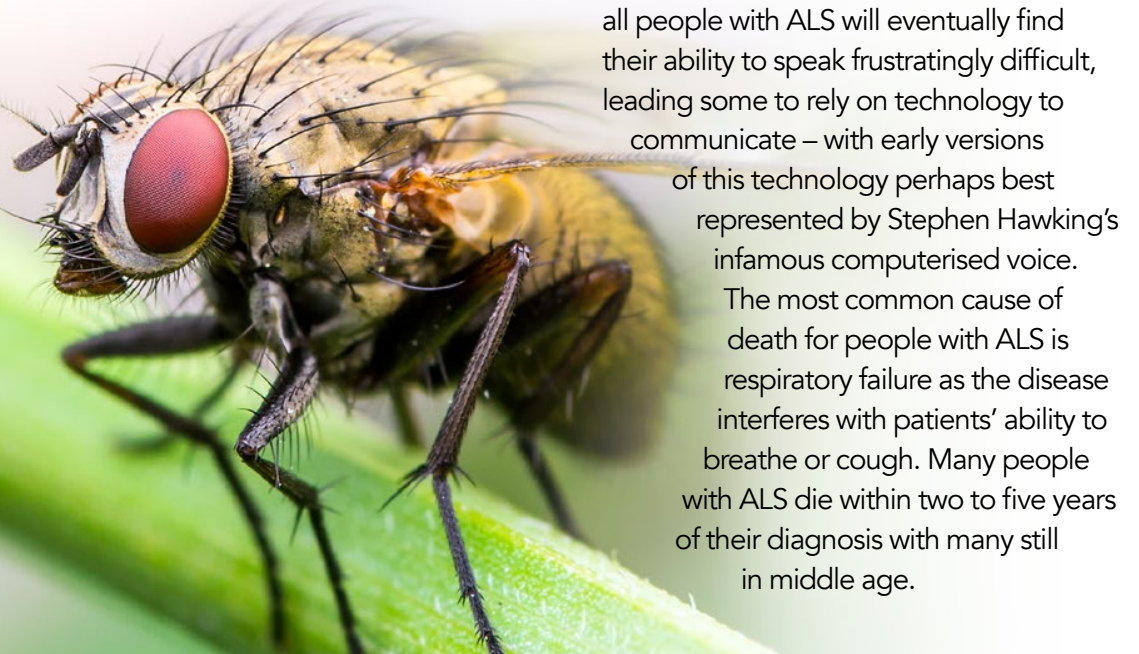

Risk factors are not well understood but include older age, with most people diagnosed in midale to older adulthood. A small proportion of cases are inherited and run in families, whilist most cases are apparently sporadic in nature. Although several genes have been linked to ALS, no one gene hat has been discovered

The urgent need for more research into ALS has gained awareness after highprofile campaigning by advocacy and patient groups. A very successful 'Ice Bucket Challenge' online campaign firmly cemented ALS in the public conscientiousness in the past years and raised over $\$ 100$ million to support vital research and services for the people living with the disease.

Despite increased awareness and funding for the condition, there is a paucity of effective treatments available for people living with the condition. After decades of research, there are currently no cures for people living with ALS. Existing treatments are limited and can help patients manage extend life by up to three months. Clinical trials for desperately-needed treatments are few and far between and relatively little is known about the molecular processes that are responsible for its widespread effects on the central nervous system.

\section{PROMISING NEW HORIZONS} Dr Leeanne McGurk, a postdoctoral esearcher is based in Dr Nancy Bonini's laboratory at the Department of Biology at the University of Pennsylvania, has committed her working life thus far to understanding more about the molecular mechanisms underlying ALS in the hope fof uncovering new avenues in the quest McGurk and Bonin treatments. Together, strides to pinpoint molecules that could

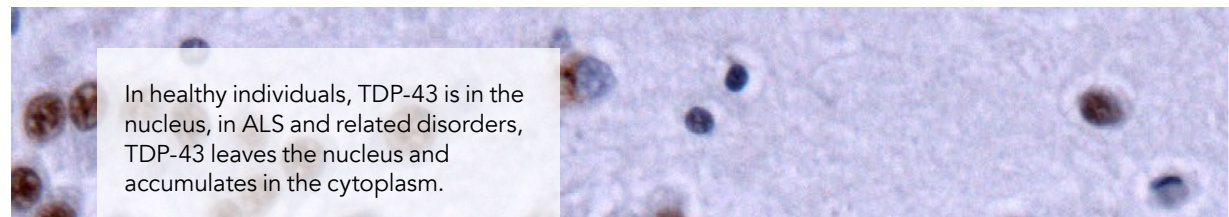
TDP-43 accumulates in the cytoplasm.
McGurk and Bonini found that under stress, disease-like TDP-43 3 accumulations for
near structures called stress granul es
Photo credit: Dr Led

help tackle some of the processes that go awry in the condition. As part
of their research tool kit, Drs McGurk and Bonini have made some of their most (undamental discoveries using a small but powerful model of neurodegenerative disease - common fruit thes. They extend their findings from the tiny ly to other systems and approaches in

\section{FRUIT FLIES TO MODEL ALS} Drosophila melanogaster-more they may first appear and have been used in scientific studies of disease for over 100 years. Fruit flies share many of the genes that are seen in humans and display more complex behaviours than may be expected, from movement to social interactions. The fact that fruit flies have a smaller genome than other laboratory animals, such as mice, means that their DNA can be studied more easily than mammals. Their shorter rather than months make itedin days and less expensive for researchers to track disease-related changes that occur over a
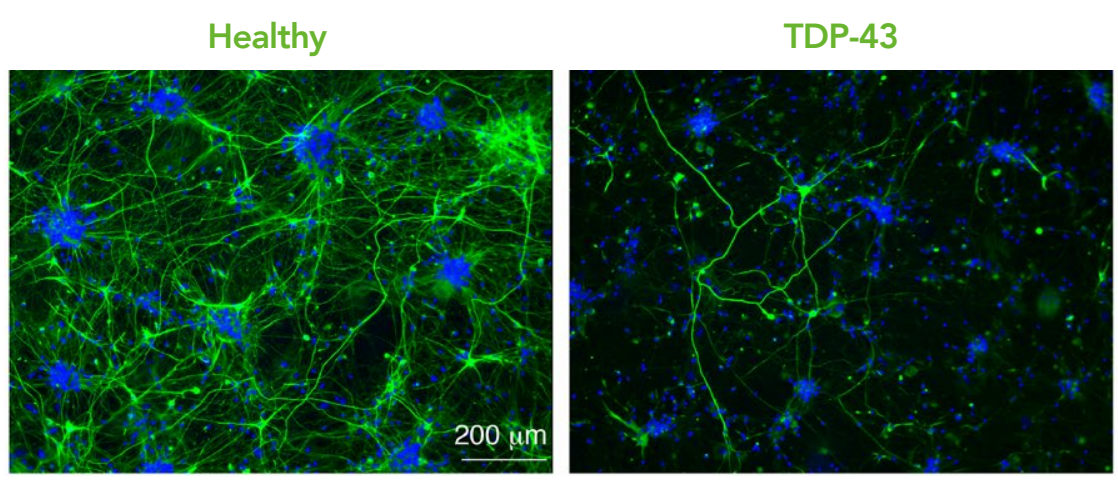

Rat spinal cord cultures which also involves the TDP-43 proten in approximately half of all cases. surprisingly more similar to humans tha

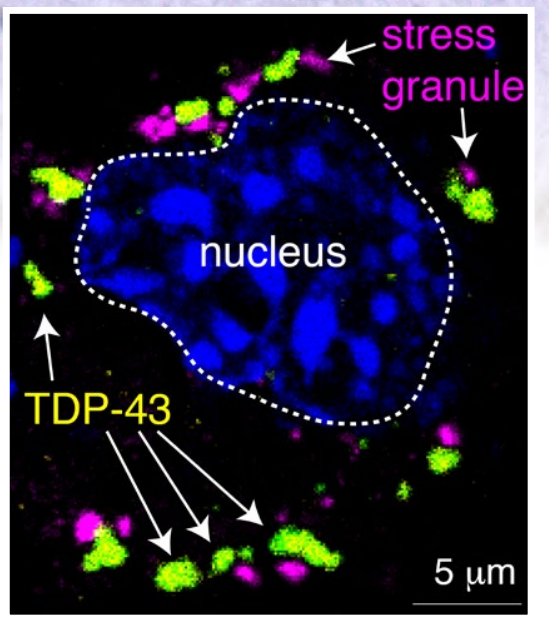

manipulating cells in the nervous system to express human TDP-43. Flies expressing this human protein showed both neurodegeneration and a shortened life
span, similar to features reflective of ALS. This advance was a vital mileston as it offered the team a model for efficiently studying ALS on the molecular level. Further studies with fruit llies shed light on the behaviour of TDP-43, focusing and tau, and frontotemporal degeneration, transportation throughout the cell and the stress, such as that seen in the disease.

\section{TDP-43 TARGETED THERAPIES} In exciting follow-up experiments, Bonini and McGurk's team found that the damage

TDP-43 + PARP inhibitor

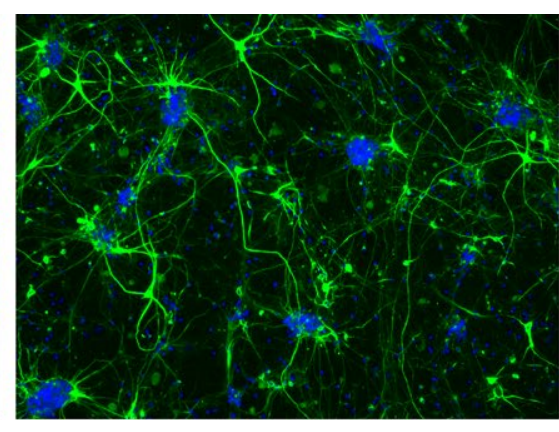

- = nucleus $\quad$ = nerve cells neural loss (middlle panel). McGurk and Bonini found that treating the nerve cells with an inhibitor to PARP-1/2 reduces the nerve cell loss caused by expression of TDP-43. This figure was previously published in L. McGurk, etal al "Nuclear Poly(ADP-Ribose) Activity Is a Therapeutic Target in Amyotrophic Lateral 


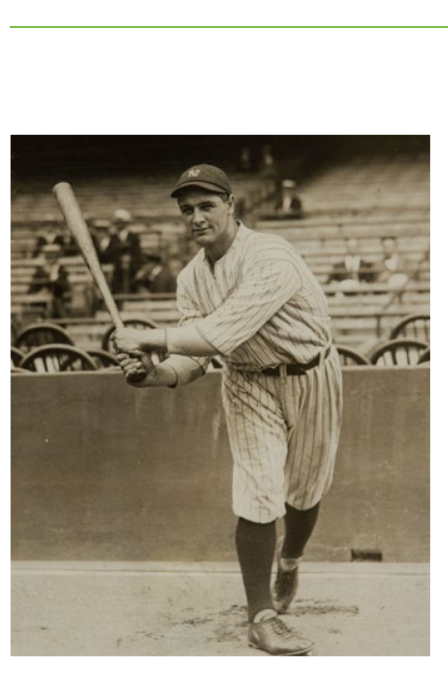

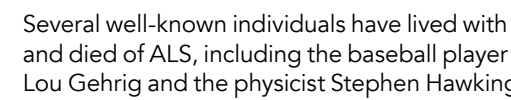
caused by expressing TDP-43 in the fly can be rescued. They discovered that reducin levels of an Thzyme known as PARP-5 stopped the ealy deth. The rosearchers believe that PARP enzymes could be key to understanding why proteins accumulate in the cell cytoplasm outside of the nucleus and further, could shed light on ways to reduce the build-up of TDP-43 in this part of the cell.

When McGurk and Bonini investigated this with their collaborators in human spinal cord cells, they found that PAR - a chain of molecules tagged on to target proteins by PARP and controls the toxicity of TDP-43 - were found in high levels in the nucleus of motor nerve cells. This suggested that levels of PARP aclity in the human spinal
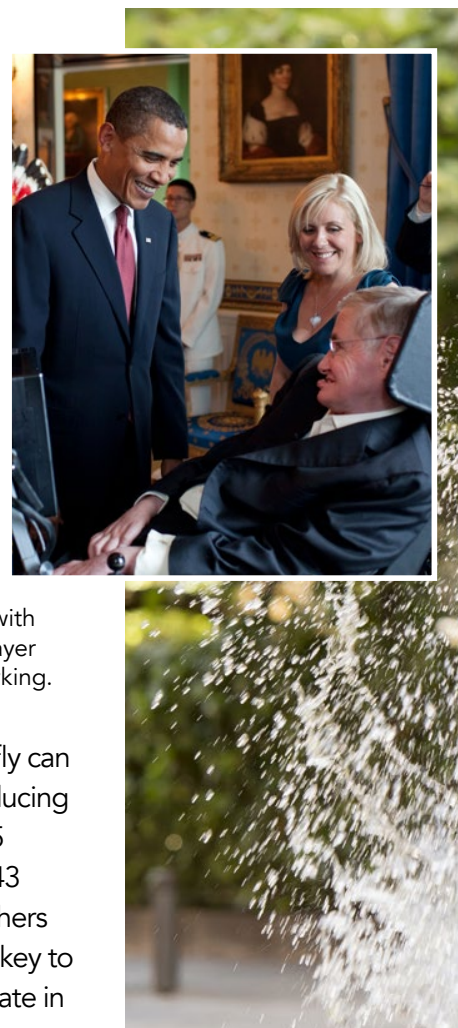

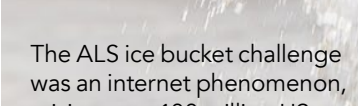
raising over 100 million US
dollars for resources and research into ALS.

To test this theory further, the researchers tested a drug to reduce activity of PARP-1/2 in a rat nerve cell model of ALS expressing human TDP-43. Promisingly, the drug - originally developed as a cancer treatment - was shown to reduce neura

Together, McGurk and Bonini have made important strides to pinpoint molecules that could help tackle some of the processes that go awry in the condition.

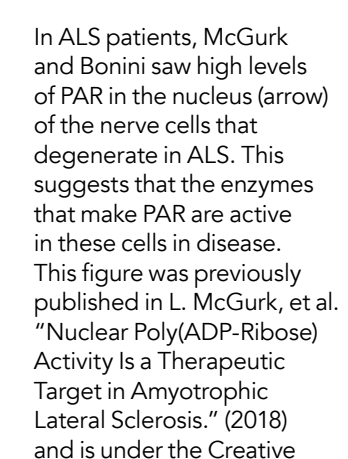

and is under the Creative
Commons Licence CC BY 4.0
Healthy

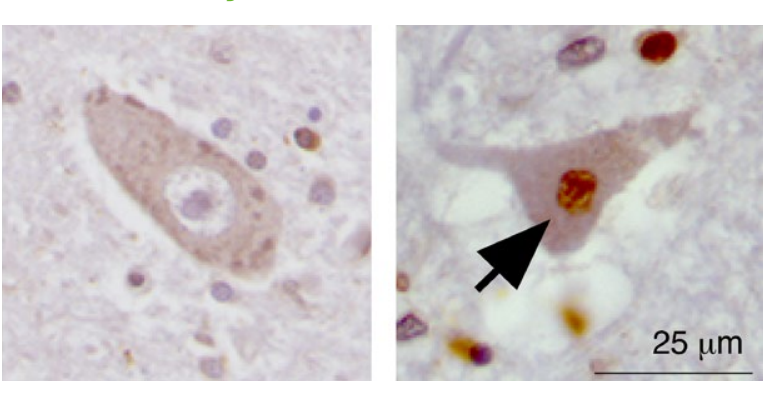

Patient motor neurons

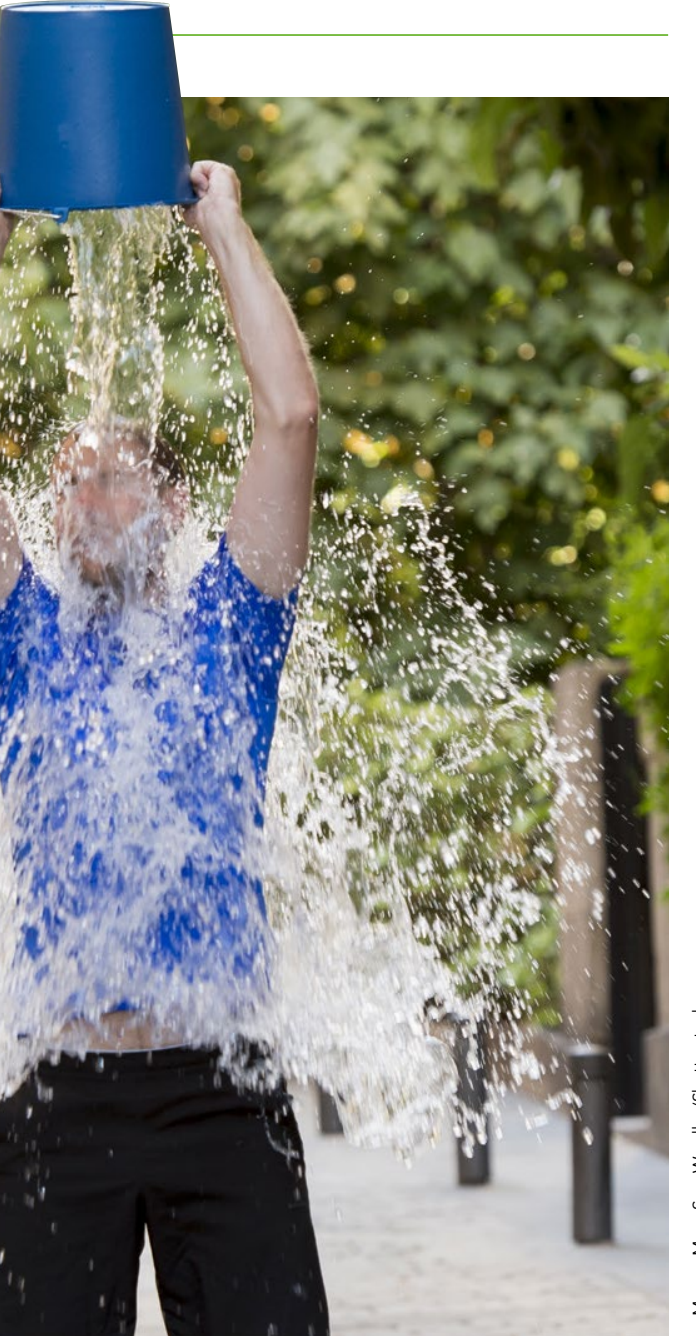

suggest that PARP enzymes could be a vital target for investigating new drug treatments in diseases linked to TDP-43 clumping

\section{FUTURE STEPS}

There is still much to learn about PARP enzymes before they could be used to and how they affect the transport of TDP-43 throughout the cell. However these findings shed light on the molecula mechanisms underlying the disease and are a beacon of hope in a drug discovery landscape blighted by a drought of advances in recent years. The findings could also shed light on treatments for disorders that share some of the same molecular characteristics, including frontotemporal degeneration. Whilst there is some way to go before it can be determined whether PARP-based therapies could work for ALS, scientists such as Drs McGurk and Bonini continue to strive towards understanding ALS at living with this devastating condition. subtypes of PARP enzymes work the the

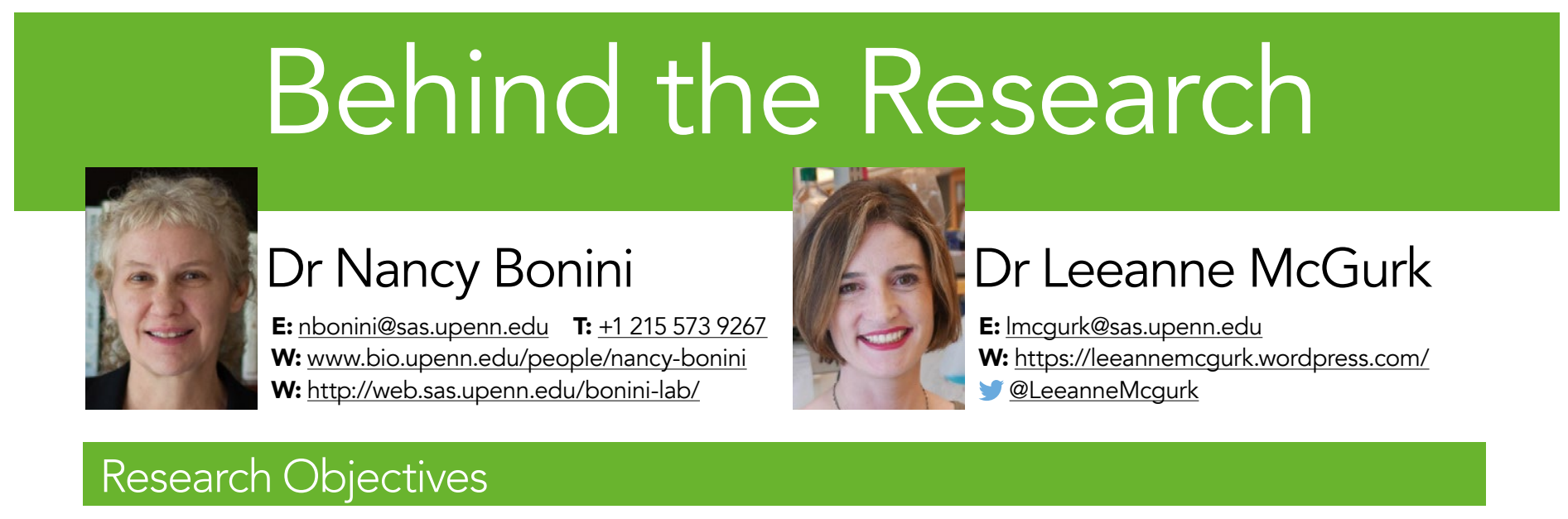

Drs Nancy Bonini and Leeanne McGurk are using fruit flies, mammalian systems and in vitro protein preparations to investigate promising new molecules that could help lead to new treatments for amyotrophic lateral sclerosis (ALS).

\section{Detail}

204G Lynch Laboratory, Department of Biology, University of Pennsylvania, Philadelphia, PA. 19104, USA

Bio

Bonini received her PhD from the University of WisconsinMadison, then performed postdoctoral research at Caltech. She then launched her laboratory at Penn, where she developed Drosophe is a model for human of the Nationative of Medicine and American Academy of Arts and Sciences

McGurk received her PhD from the University of Edinburg and joined Bonini at Penn as a National Ataxia Foundation Investigator. As a post-doctoral researcher, she uncovered that the post-translational modification poly(ADPribosylation) may play a role in mediating amyotrophic lateral sclerosis. This coming Fall, she wil join the Division of Cet \& University of Dundeg, as an independent investigas

\section{Funding
NIH/NINDS}

\section{Collaborators}

Investigators at Penn:

- DrVivianna Van Deerl

- Dr Edward B Lee

- Dr John Q. Trojanows

- Dr James Shorter

An investigator at No

- Dr Robert G. Kalb

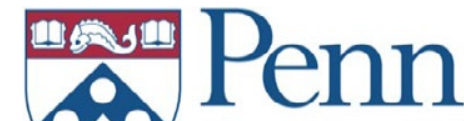 Arts E'Sciences}

\section{References}

McGurk, L. et al (2015). 'Drosophila as an In Vivo Model for Human Neurodegenerative Disease'. Genetics, 201 (2): 377-402. McGurk, L. et al (2018). 'Poly(ADP-Ribose) Prevents Pathological Phase Separation of TDP-43 by Promoting Liquid Demixing and
Stress Granule Localization'. Molecular Cell, 71(5):703-717. McGurk, Let al (2018), 'Nuclear poly(ADP-ribose) activity is a therapeutic target in amyotrophic lateral sclerosis'. Acta Neuropathol Commun, 6(1):84.

McGurk, L. et al (2018). 'Poly(ADP-ribose) engages the TDP-43 nuclear-localization sequence to regulate granulo-filamentous aggregation'. Biochemistry, 57 (51), 6923-6926.

\section{Personal Response}

What steps would have to be taken before PARP enzyme-based therapies could be tested in a clinical
trial?

II ALS is a heterogeneous disease, meaning that there are many different genes that when mutated give rise
to the disease. We need to understand if PARP inhibitors are beneficial to all, or only some of these disease gene situations using our mode systems of cells and animals. These types of studies will inform if certain patients may promising. Many of the developed PARP inhibitors have very specific modes of action and some are better at crossing the blood-brain-barrier than others. We need to first understand if there are differences in these PARP inhibitors in treating ALS-associated toxicity in cells and compounds for further testing. Traditionally, drugs are tested in rodent models of disease before they can move forward into a clinical setting. The field has made much progress in rodent models that recapitulate aspects of the disease process. Once we know which inhibitors are most promising in our settings, the next step will be to advance and test 